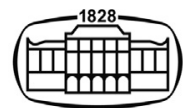

AKADÉMIAI KIADÓ

\title{
Global bioethical challenges of medicalising psychedelics
}

\section{RICCARDO MICELI MCMILLAN ${ }^{1,2 *}$ (1)}

\section{Journal of Psychedelic Studies}

5 (2021) 2, 57-64

DOI:

$10.1556 / 2054.2021 .00188$

(c) 2021 The Author(s)
ORIGINAL RESEARCH PAPER

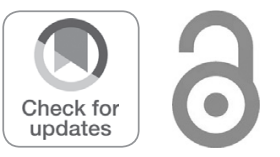

*Corresponding author. E-mail: Riccardo.micelimcmillan@ uqconnect.edu.au

\author{
${ }^{1}$ University of Queensland, Faculty of Medicine, Australia \\ ${ }^{2}$ University of Sydney, Faculty of Medicine and Health, Australia
}

Received: July 13, 2021 • Revised manuscript received: August 26, 2021 • Accepted: September 1, 2021

Published online: September 15, 2021

\begin{abstract}
Psychedelic-assisted psychotherapy is an emerging psychiatric treatment that is attracting significant scientific, medical, and public attention. Whilst preliminary results from empirical studies are promising, the medical use of these compounds is highly controversial. Surprisingly, and despite the current controversies caused by the re-medicalisation of psychedelics, bioethicists have remained mysteriously silent. This paper aims to stimulate further bioethical reflection regarding the re-medicalisation of psychedelics. The current paper aims to do this by applying a normative phenomenological lens of analysis. Namely, this paper applies Martin Heidegger's critique of modern technology, and Fredrik Svenaeus' extension of this critique, to the re-medicalisation of psychedelics. I argue that when this critique of modern technology is applied several normative issues become apparent. Specifically, it becomes apparent that the re-medicalisation of psychedelics risks turning the ecological sources, cultural contexts, and experiences induced by psychedelics into resources to be exploited for human goals; all of which risks endangering ecosystems, appropriating traditional knowledge, and reducing the therapeutic effects of psychedelic-assisted psychotherapy. Furthermore, I suggest that preserving nonreductionist, non-instrumentalising traditional ways of understanding psychedelic compounds is essential in mitigating these consequences. More discussion by bioethicists is necessary as these consequences represent important global challenges for the psychedelic renaissance that require immediate addressing.
\end{abstract}

\section{KEYWORDS}

psychedelics, phenomenology, bioethics, ethics, hallucinogens

\section{INTRODUCTION}

The term 'psychedelic' refers to a range of hallucinogenic compounds that induce altered states of consciousness (Garcia-Romeu, 2016). Broadly speaking, these compounds can be categorised into four classes: classical psychedelics (e.g. Psilocybin, LSD, DMT), entactogens (e.g. MDMA), dissociative anaesthetics (e.g. Ketamine), and atypical hallucinogens (e.g. Ibogaine). Each of these different classes are characterised by unique pharmacological mechanisms, as well as unique experiences or "altered states" (Garcia-Romeu, 2016).

Psychedelic-assisted psychotherapy is currently being explored as a treatment for a variety of mental illnesses (Reiff et al., 2020). The majority of current research focusses on the use of MDMA-assisted psychotherapy for the treatment of post-traumatic stress disorder and Psilocybin-assisted psychotherapy for the treatment of major depressive disorder. However, other disorders currently under investigation include end-of-life distress, substance-use disorders, body-identity disorders, and obsessive-compulsive disorder (Reiff et al., 2020).

The use of psychedelics to treat mental illness is promising, with preliminary studies suggesting these medicines could be more efficacious (Davis et al., 2021) and cost-effective (Marseille, Kahn, Yazar-Klosinski, \& Doblin, 2020) than current standards of care. However, these re-emerging medicines are also controversial. Whilst the controversial nature of these compounds is in part due to non-medical factors that existed at the time of their initial 
integration into Psychiatry (Rucker, 2015), it cannot be disputed that these compounds induce noteworthy changes in human cognition. As such, it is imperative that bioethicists play a role in guiding the use of these compounds. Concerningly, to date there is a paucity of literature published in bioethics journals regarding ethical considerations brought about by psychedelic medicalisation. For example, the BMJ's Journal of Medical Ethics - arguably the bioethics journal with the highest impact in the field (Bioethics Research Library, 2016) - has at the time of writing this manuscript only three publications relating to psychedelic bioethics (Miceli McMillan, 2020; Smith \& Sisti, 2020; Strauss, de la Salle, Sloshower, \& Williams, 2021). The relative lack of research output from an academic discipline that aims to investigate normative issues relating to medical and life sciences (Chadwick, 2019) is surprising.

In this paper I use a phenomenological approach in order to reveal some normative consequences of medicalising psychedelics. I have chosen a phenomenological lens in light of empirical findings demonstrating that the efficacy of psychedelic-assisted psychotherapy is predicted by the subjective experiences (e.g. "mystical-like" experiences) induced by these compounds (Roseman, Nutt, \& CarhartHarris, 2018). In other words, the therapeutic effects of psychedelics are mediated by the kinds of first person experiences patients have; and are not purely due to neurochemical phenomena. Therefore, phenomenology (i.e. "the study of structures of consciousness as experienced from the first-person point of view" (Smith, 2018)) provides a useful approach as it is a non-reductionist approach that values the primacy of subjective experience.

In this paper I apply Martin Heidegger's (1977) critique of modern technology, as well as Fredrik Svenaeus' (2017) extension of this critique, to psychedelics. In doing so, I argue that the re-medicalisation of psychedelics risks turning the ecological sources, cultural contexts, and experiences induced by psychedelics into resources to be exploited for human goals. I suggest that this instrumentalisation brought about by psychedelic medicalisation could lead to the endangerment of ecosystems, erasure of traditional knowledge, and reduction in the therapeutic effects of psychedelicassisted psychotherapy; all of which are consequences that bioethical guidance and reflection could assist in mitigating.

\section{MARTIN HEIDEGGER'S CRITIQUE OF MODERN TECHNOLOGY}

In his essay "The Question Concerning Technology", Heidegger sets out to define the essence of modern technology (Heidegger, 1977). On his account, modern technology can be understood as a "mode of revealing". That is to say, what defines modern technology is that it changes the experience of the world, or in Heideggerian language, it reveals particular meaning patterns in the world (Heidegger, 1977, p. 12). The next question which Heidegger addresses is what kind of change in the meaning patterns of the world does modern technology produce? Modern technology, according to Heidegger, reveals the world as a Standing-Reserve. That is to say, modern technology reveals the world as a resource to be used for human goals (Heidegger, 1977, p. 17). Furthermore, by changing the meaning patterns of the world and revealing the world as a Standing-Reserve, modern technology also alters the pursuits which are experienced as being worthwhile (Svenaeus, 2017).

To illustrate how modern technology reveals the world as a resource - and the consequences that this revealing entails with regards to guiding human pursuits - Heidegger draws on the example of a hydroelectric plant on the Rhine river (Heidegger, 1977, p. 16). He argues that the construction of the hydroelectric plant on the Rhine changed how the river was experienced such that it was no longer revealed as just a river; it became revealed as a resource to be used for human ends, a mere source of energy (Heidegger, 1977, p. 16). This change in experience of the river entails an inevitable change in how humans interact with it (i.e. what pursuits are worthwhile with regards to the river).

Heidegger argues that the mode of revealing which defines modern technology is not necessarily problematic in of itself. What becomes problematic is when this resourcedriven mode of revealing dominates and becomes the only mode of revealing. According to Heidegger, modernity is defined by a domination of the modern technological way of revealing, a phenomenon he terms Enframing (or Gestell) (Heidegger, 1977, p. 20). Heidegger finds the Enframing characteristic of modernity problematic because the pervasiveness of this mode of revealing renders it invisible. Not only are other modes of revealing (and their associated benefits) lost, but the very knowledge that other modes of revealing exist is also lost; thus, putting other ways of experiencing the world out of reach and confining experience to a myopic resource-driven gaze (Heidegger, 1977; Svenaeus, 2017).

\section{HEIDEGGER'S CRITIQUE AND MEDICINE}

Fredrik Svenaeus extends Heidegger's concept of Enframing to medicine by arguing that medical technologies risk Enframing human beings. That is to say that medical technologies risk changing how human beings are experienced such that they are no longer experienced as other beings but as material resources (i.e. a Standing-Reserve) (Svenaeus, 2017, pp. 75-96). Transforming the understanding of other beings from a being-in-the-world to a material resource presents significant concerns. This is because the Enframing of other beings entails a loss in the ability to reveal other beings as experiencing subjects. In other words, the very ability to experience others as beings becomes lost from humanity's phenomenological repertoire. Instead, other beings are experienced as purely material entities which are to be used for human goals (Svenaeus, 2017, pp. 75-96). This mode of revealing which transforms beings into a Standing-Reserve entails not only changes in how beings are 
understood, but also an inevitable change in how other beings are treated.

Svenaeus goes on to apply Heidegger's critique of modern technology directly to Psychiatry (Svenaeus, 2017, pp. 75-96). Svenaeus argues that the process of Enframing in Psychiatry raises further concerns. As psychiatric technologies (e.g. neuro-pharmaceuticals) are aimed at the structures of experience themselves, and not just at experiences of the body, Svenaeus suggests that what can be Enframed in Psychiatry is the experience of being itself. In other words, because the focus of psychiatric technologies is to alter the structures of experience, Enframing in this context entails directing the resource-driven gaze of modern technology onto one's own structures of experience (Svenaeus, 2017, pp. 75-96).

As Heidegger explicated, what is dangerous about the mode of revealing which defines modern technology is not how it reveals the world, but rather the dominating nature with which it replaces other ways of revealing the world (Heidegger, 1977; Svenaeus, 2017). Therefore, according to Svenaeus, the solution to the dangers of Enframing in medicine is to support the revealing of the world in other ways which prioritise the primacy of experience (i.e. phenomenology). In doing this, medicine can benefit from the advantages which modern technology has provided without leading to the consequences of Enframing human beings (Svenaeus, 2017, pp. 75-96).

\section{ENFRAMING OF PSYCHEDELIC PLANTS}

As psychedelics become re-medicalised, the way in which these compounds are understood is changing rapidly. I propose here that as psychedelics undergo the process of becoming an official medicine (i.e. a medical technology), their ecological sources are becoming Enframed. That is to say that the process of psychedelics becoming approved medicines is causing the ecological sources of these compounds to become understood only as material resources which can be harvested for human goals, similar to how mining companies understand precious stones and minerals. The revealing of these ecological sources as a StandingReserve (i.e. a resource to be used) presents several normative challenges.

As psychedelics have regained popularity, the ecological sources of these compounds have become increasingly at risk of unsustainable harvesting practices. Now that many psychedelics sit at the brink of becoming approved medicines (MAPS, 2021) - a change which will create an enormous demand for these compounds - their ecological sources face an unprecedented level of vulnerability.

Concerns regarding unsustainable harvesting of psychedelics have been voiced consistently in psychedelic-using communities (David, 2017; Ermakova \& Terry, 2020; Ermakova et al., 2020; Francuski, 2020; Nickles \& Nickles, 2014; Psychedelic Community, 2018; Siebert, 2021a, 2021b; Tomoski, 2019; Virdi, 2020). Debates regarding ecologically destructive harvesting driven by non-medical psychedelic use have been raised with regards to Mescaline (David, 2017; Ermakova \& Terry, 2020; Ermakova et al., 2020; Virdi, 2020), with the ecological consequences becoming so devastating that the Peyote cactus - a source of Mescaline for traditional healers - is considered endangered by several organisations (Ermakova et al., 2020) and traditional healers are increasingly unable to access the cactus (David, 2017). Similar debates have also occurred with regards to DMT (Nickles \& Nickles, 2014; Psychedelic Community, 2018; Francuski, 2020). Namely, several of DMT's ecological sources such as the 'Ayahuasca vine', the Chacruna plant, and several Acacia subspecies have become increasingly scarce due to unsustainable harvesting practices (Francuski, 2020; Nickles \& Nickles, 2014; Psychedelic Community, 2018). With regards to 5-MeO-DMT there has been particular alarm, as the ecological source is not a plant but an animal (i.e. the Bufo Alvarius toad) (Siebert, 2021a, 2021b; Weil \& Davis, 1994). All of these concerns have in turn catalysed discussions in psychedelic-using communities about the use of synthetically derived versions of naturally occurring psychedelics (Siebert, 2021a, 2021b).

However, the use of synthetically derived versions of naturally occurring psychedelics as a potential solution to increasingly unsustainable demand is a topic which is not uncontroversial (Labate, 2020; Tomoski, 2019). A full analysis of debates regarding differences between using synthetically derived psychedelics vs. plant-based psychedelics is beyond the scope of the current manuscript. However, to provide readers with some context, common discussion points that arise against the use of synthetic psychedelics include the loss of unrecognised therapeutic compounds in the host plant (Tomoski, 2019) and the loss of potentially therapeutic rituals involved in the preparation and consumption of a host plant (Labate, 2020). Therefore, whilst synthetically-derived psychedelics might provide a solution to the aforementioned concerns regarding unsustainable harvesting of sacred plants, the medicinal use of synthetic psychedelics raises a host of further questions relevant for bioethicists regarding how best psychedelic compounds and the rituals they are housed in should be integrated into modern psychiatric practice.

The ecological source of Psilocybin (i.e. Psilocybe genus of mushroom), has not been subject to the same level of environmental concern as other psychedelics, likely due to its' relative abundance and the fact that mushrooms grow much faster than other psychedelic sources. However, despite these facts, instances where there have been sudden, large increases in demand for Psilocybe mushrooms have been reported to cause scarcity for traditional healers (Darnell, 2021; Harvey, 2020). Therefore, as Psilocybin leads the charge in the medicalisation of psychedelics, it will be important to track the ecological consequences of turning Psilocybe mushrooms into an exploitable resource.

Interestingly, non-official guides to sustainable harvesting of other botanical medicines sometimes include advice such as pausing and showing gratitude towards the ecological source of the medicine (Milson \& Pratt, 2017). This advice is also paralleled by the language used to describe the 
ecological sources of psychedelics by traditional healers and other psychedelic-using communities. The ecological sources of psychedelics are not viewed as resources, they are gifts from god (David, 2017), sacred gateways to connect with divine spirits (Gearin, 2017; Labate \& Cavnar, 2014), or living beings (Labate, 2020); as such, these sources require an extreme amount of respect. In other words, sustainable harvesting and use of psychedelic sources, entails revealing these sources in non-resource dominating ways. Therefore, if these sources are to be protected, a potential solution could come from learning/fostering how traditional healers and other psychedelic-using communities understand the ecological sources of psychedelics.

\section{ENFRAMING OF PSYCHEDELIC CULTURES}

The aforementioned shift in how psychedelic sources are being understood raises another normative concern. Turning the resource-gaze onto the ecological sources of psychedelics also raises concerns regarding the treatment of traditional communities in which psychedelic healing rituals have been developed. The shift towards understanding psychedelic sources as resources, could also entail that traditional communities which use psychedelics become understood as resources; a phenomenon which has already caused debate due to surges in 'psychedelic tourism' (Lagarde, 2021; Negrin, 2020). In other words, the medicalisation of psychedelics, not only Enframes the ecological sources of psychedelics, but also the cultural contexts in which therapeutic uses of psychedelics have been developed.

The history of Psilocybin would suggest that concerns regarding the treatment of traditional psychedelic-using communities are not unfounded, as the introduction of Psilocybin to the West brought with it a paradigmatic example of the destructive effects that Enframing has on traditional communities and practices. The story of María Sabina (de Lima, 2014; Gerber et al., 2021) is often recounted as a warning about the destructive effects that the commercialisation of psychedelics could have on traditional healers and serves as an invaluable case study from which bioethicists could learn. María Sabina was a traditional Mazatec healer who used Psilocybin containing mushrooms in traditional healing rituals. As a traditional healer she was highly regarded by her community (de Lima, 2014; Gerber et al., 2021). However, following her encounter and ceremonies with Robert Gordon Wasson - an American banker, ethnomycologist, and author - Sabina faced significant challenges (de Lima, 2014; Gerber et al., 2021). Wasson published a now infamous piece entitled "Seeking the Magic Mushroom" in Life magazine, detailing his privileged experience as one of the first "white men in recorded history to eat the divine mushroom" or as his article coined, the "magic mushroom" (Wasson, 1957). Despite promises to keep Sabina's identity anonymous, Wasson later published works which contained photographic depictions of Sabina and her work. The widespread interest caused by Wasson's work, along with Sabina being identifiable, resulted in an unprecedented influx of foreigners seeking Sabina and her rituals (de Lima, 2014; Gerber et al., 2021). This led to what Sabina termed, the "desacralisation" of the mushroom and also led to Sabina facing significant repercussions from her local community for allegedly exploiting the secrets of the sacred ritual; including having her house burnt down and being imprisoned (de Lima, 2014; Gerber et al., 2021).

How traditional psychedelic-using communities and their knowledge can be integrated into modern Psychiatry without being colonised is a topic which imminently needs addressing, and it is reassuring to see it recently being raised by scientific circles (Gerber et al., 2021). Understanding the medicalisation of psychedelics as a kind of Enframing could provide a useful lens to help move this discussion forward. As was mentioned earlier, Enframing is damaging because it dominates and displaces other ways of revealing the world. Therefore, fostering and encouraging other modes of revealing provides a potential solution which mitigates the damage Enframing could cause. Whilst this understanding does not necessarily provide a concrete path forward, it highlights how the path must include substantial input from traditional healers and other psychedelic-using communities. Without incorporating other modes of revealing found in psychedelic-using communities, these communities, their knowledge, and their medicines, are at risk of becoming Enframed - a fate which could have disastrous ecological and cultural consequences.

\section{ENFRAMING OF PSYCHEDELIC EXPERIENCES}

I also wish to propose here that as psychedelics undergo the process becoming medical technologies, not only do their ecological sources and cultural contexts become Enframed, but so do the kinds of experiences which they induce. That is to say that psychedelic states of consciousness become understood only as a resource which can be used for human goals (i.e. treating mental illness). I will argue that the Enframing of psychedelic states of consciousness has tangible, detrimental therapeutic and nontherapeutic implications.

Given medicine's often reductionist tendencies (Beresford, 2010), as psychedelic experiences become understood purely as a medical resource, these experiences are likely to become reduced to neurochemical resources. That is to say that psychedelic experiences (and their utility for human goals) are at risk of being explained at purely a neurochemical level of analysis. For example, "mystical-like" experiences and their therapeutic effects will become understood purely with reference to disruptions of neural networks or alterations in neurotransmitters. In losing other non-reductionist modes of revealing psychedelic experiences, I argue that the effectiveness of psychedelic-assisted psychotherapy will be diminished. This is because the reduction of psychedelic experiences to a neurochemical Standing-Reserve, entails an inextricable erosion of the value that psychedelic experiences hold in of themselves. 
The reduction of psychedelic experiences into a neurochemical resource is arguably already occurring and leading to consequences such as the development of what have been called "Second Generation Psychedelics" (compounds which attempt to alter the same neurochemistry as psychedelics without inducing altered state experiences (Rosner, 2020; Siebert, 2021a, 2021b) ${ }^{1}$ ). Empirical literature has demonstrated that the psychedelic experience itself (Roseman et al., 2018), as well as many psychosocial factors surrounding psychedelic experiences (e.g. physical settings and music) (Hartogsohn, 2016), play key roles in determining therapeutic outcomes. The Enframing of psychedelic experiences I suggest is already underway and has initiated the process of reductionist modes of revealing dominating over nonreductionist modes of revealing. This domination of reductionist ways of understanding psychedelic experiences will by its very nature lead to a displacement of non-reductionist modes of understanding; modes of understanding that often stem from traditional psychedelic-using communities and centuries of accumulated knowledge. As non-reductionist ways of revealing psychedelic experiences become displaced, and these experiences become understood only in terms of neurochemistry, it is likely that the therapeutic effects of psychedelic experiences will diminish. This is because much of the therapeutic benefit of psychedelics seems to be derived from non-neurochemical aspects of these experiences, aspects which have been nurtured by psychedelic-using communities and their non-reductionist modes of revealing psychedelic experiences. Therefore, I argue that understanding psychedelic experiences only as neurochemical aberrations which can be used to treat mental illness brings with it the danger of concealing other modes of understanding that are likely essential in producing therapeutic outcomes.

However, it is important to note here that non neuroreductionist modes of understanding also display the potential to Enframe psychedelic experiences. For example, psychological and holistic approaches that understand 'mystical-like' experiences as only a vehicle through which to attain mental well-being risk concealing other non-therapeutic modes of understanding these experiences. This Enframing of psychedelic experiences as only therapeutic resources to be used for the treatment of mental illness raises some non-therapeutic concerns. For example, in losing nontherapeutic ways of understanding psychedelic experiences, how might other potential applications of these experiences become concealed? Research demonstrates that psychedelics can engender profound, life altering experiences (Roseman et al., 2018). In understanding these experiences only as therapeutic resources, is the power of such experiences being held hostage by the medical model? How psychedelic experiences are understood will inevitably shape the

${ }^{1}$ In fairness, other emerging therapeutic applications of psychedelics such as the treatment of primary headache disorders (Andersson, Persson, \& Kjellgren, 2017), might benefit from Second Generation Psychedelics (having to trip every time you have a migraine might not be overly functional). endeavours which are pursued through their use. Therefore, preserving different ways of understanding psychedelic experiences will elucidate other ways in which we should, at least consider, using psychedelics in society. For example, shamanic modes of revealing psychedelic experiences might suggest that these experiences represent connections with the divine; psychonautic (Móró, 2011) modes of revealing psychedelic experiences might suggest that these experiences represent a form of deep self-knowledge; recreational modes of revealing psychedelic experiences might suggest that these experiences represent intrinsically pleasurable states.

Interestingly, resistance to the Enframing of psychedelic experiences, and an associated attempt to preserve traditional (and often non-instrumentalising) modes of revealing psychedelic experiences, has recently found expression in the aptly named Sabina Project (Adams, 2020; Darnell, 2021; The Sabina Project, n,d.). The founders of the Sabina Project state that they aim to resist the process of psychedelic colonisation by integrating ancestral plant wisdom into modern psychedelic experiences (The Sabina Project, n,d.). Another similar endeavour can be found at the Chacruna Institute for Psychedelic Plant Medicines (Labate, 2020). The Chacruna Institute claims that it builds bridges between traditional use of psychedelic plants and modern psychedelic science (Labate, 2020).

Such projects are vital and require more institutionallevel support. Without conservation of traditional modes of revealing psychedelic experiences, not only will psychedelics become colonised, they will also become Enframed - a fate which would entail losing many of the benefits (therapeutic and otherwise) that psychedelic experiences could provide.

While psychedelic traditions emerged from a variety of civilizations-including Bwiti, Amazonian, Aztec, Mazatec, Mayan, and Incan cultures-those cultures are rarely recognized today in psychedelic research and medicine, even though psychedelics are going through somewhat of a renaissance. (Adams, 2020)

\section{EXISTING GLOBAL BIOETHICS DEBATES AND PSYCHEDELIC ENFRAMING}

Interestingly, and of particular relevance for bioethicists interested in pursuing the normative issues raised in the foregoing, there is an existing literature that aims to address the ethical challenges brought about by the commercialisation of traditional medicines. Namely, the International Bioethics Committee (2012) prepared a report addressing the ethical implications of traditional medicine systems. In this report, the International Bioethics Committee addressed a number of areas of concern.

One area of concern raised by the International Bioethics Committee (2012) with regards to the commercialisation of traditional medicines was Biopiracy. Biopiracy is defined as "the unjustified use of intellectual property mechanisms, in order to justify the exclusive appropriation over multiple resources, products, and biological processes that have been 
used, in some cases, through indigenous knowledge." (Ramirez Garcia, 2014). Bioethics literature on Biopiracy is extensive (Ramirez Garcia, 2014) and argues that not only can this process lead to the dispossession of communities and their knowledge (International Bioethics Committee, 2012), but as illustrated in the examples with psychedelic plants above, Biopiracy leads to scarcity of medicinal plants for local communities (Mackey \& Liang, 2012). Future work in bioethics should explore how developments in the literature on Biopiracy such as "benefit sharing" legal frameworks (Hoffmann, 2015) could help mitigate the potential cultural and ecological consequences of Biopiracy specifically in the context of psychedelic medicalisation.

Another area of concern raised by the International Bioethics Committee (2012) was how researchers ought to balance the often competing moral imperatives of improving scientific knowledge regarding effective healthcare while also protecting the traditional health practices from which this knowledge stems. In regards to this dilemma the International Bioethics Committee (2012) states:

Local communities which have been handing down traditional practice for generations and have a detailed knowledge of the logic on which it is based are best placed to contribute to informed, sustainable use of biological resources and to protect their own identity and well-being. On the other hand, governments and the international community should regard everyone's access to the indisputable and unprecedented opportunities offered by modern medicine as a moral and political obligation...

\section{(International Bioethics Committee, 2012, p. 18)}

As was argued in the foregoing, preserving traditional ways of revealing psychedelic rituals and experiences is necessary to stop these phenomena becoming Enframed. However, as the International Bioethics Committee (2012) highlights, this preservation ought to be balanced with an obligation to ensure that the international community has access to knowledge about medications that restore health. How best traditional knowledge of psychedelic practices and modern psychiatric knowledge of psychedelic practices can be integrated to effectively balance these moral obligations is an immensely important topic that warrants bioethical input. However, it is imperative that this input carefully considers the power imbalances that might arise when addressing this dilemma. Bioethics work that accounts for these power differentials via fair consultation processes that involve the various parties at stake is urgently needed.

\section{CONCLUSION}

The re-emergence of psychedelics in medicine presents a unique opportunity to address an epidemic of suffering in society. This opportunity can no longer be ignored in good faith. However, this opportunity also presents a minefield of potentially catastrophic consequences. Bioethicists must start paying more attention. Their mysterious absence in guiding one of the most potentially important and powerful emerging medical technologies should be a source of grave concern.

In this paper I have attempted to catalyse the process of bioethical reflection on psychedelic medicalisation. In order to do this, I used a phenomenological lens to reveal normative concerns regarding psychedelic re-medicalisation. Namely, I applied Martin Heidegger's critique of modern technology, as well as Fredrik Svenaeus' extension of this critique, to the emerging psychedelic renaissance in medicine. In doing so, I argued that the re-medicalisation of psychedelics risks turning the ecological sources, cultural contexts, and experiences induced by psychedelics into resources to be exploited for human goals; all of which could lead to the endangerment of ecosystems, erasure of traditional knowledge, and reduction in the therapeutic effects of psychedelic-assisted psychotherapy. Furthermore, I suggested that the preservation of traditional non-reductionist, non-instrumentalising modes of revealing psychedelics is essential in preventing these outcomes. More input is imminently needed to address these global concerns. I hope that by raising these issues here, more discussions surrounding how the psychedelic renaissance can proceed responsibly will follow.

Funding: N/A.

Competing interests: N/A.

Ethics approval statement: N/A.

Contributorship statement: Riccardo Miceli McMillan was the sole author of this manuscript.

\section{ACKNOWLEDGEMENTS}

N/A.

\section{REFERENCES}

Adams, B. (2020). Psychedelic roots: The Sabina project aims to restore BIPOC sacred earth medicine [Internet]. Forbes. Available from: https://www.forbes.com/sites/benjaminadams/ 2020/11/04/psychedelic-roots-the-sabina-project-promotesbipoc-representation-in-the-psychedelic-community/?sh $=11 \mathrm{bc}$ 0b22d100.

Andersson, M., Persson, M., \& Kjellgren, A. (2017). Psychoactive substances as a last resort-a qualitative study of self-treatment of migraine and cluster headaches. Harm Reduction Journal, 14(1), 60. https://doi.org/10.1186/s12954-017-0186-6.

Beresford, M. J. (2010). Medical reductionism: Lessons from the great philosophers. QJM: An International Journal of Medicine, 103(9), 721-724. https://doi.org/10.1093/qjmed/hcq057.

Bioethics Research Library. (2016). Top 50 bioethics journals and top 250 most cited bioethics articles since 2011, 2016 edition 
[Internet]. Bioethics Research Library at Georgetown University. Available from: https://repository.library.georgetown.edu/ bitstream/handle/10822/1043487/Top\%2050\%20Bioethics\% 20 Journals\%20 and $\% 20$ Top $\% 20250 \% 20$ Most $\% 20$ Cited $\%$ 20Bioethics\%20Articles\%20Since $\% 202011 \% 2$ c\%202016\% 20Edition\%20-\%20Bioethics\%20Research\%20Library.pdf? sequence $=1$ \&isAllowed $=\mathrm{y}$.

Chadwick, R. T. (2019). Bioethics. In: Encyclopedia Britannica [Internet]. Available from: https://www.britannica.com/topic/ bioethics.

Darnell, T. (2021). How this black-led psychedelic collective is combining anti-racism with 'sacred plant medicine' [Internet]. VOGUE. Available from: https://www.vogue.com/article/ sabina-project-psychedelic-collective.

David, D. (2017). How my elder's sacred peyote is disappearing [Internet]. Chacruna Institute for Psychedelic Plant Medicines. Available from: https://chacruna.net/my-elders-sacred-peyoteis-disappearing/.

Davis, A. K., Barrett, F. S., May, D. G., Cosimano, M. P., Sepeda, N. D., Johnson, M. W., et al. (2021). Effects of psilocybin-assisted therapy on major depressive disorder: A randomized clinical trial. JAMA Psychiatry, 78(5), 481-489. https://doi.org/10.1001/ jamapsychiatry.2020.3285.

de Lima, M. (2014). The case of María Sabina. In The Ethnopoetics of shamanism (pp. 125-160). New York: Palgrave Macmillan US.

Ermakova, A., \& Terry, M. A. (2020). A word in edgewise about the sustainability of Peyote [Internet]. Chacruna Institute for Psychedelic Plant Medicines. Available from: https:// chacruna.net/a-word-in-edgewise-about-the-sustainability-ofpeyote/.

Ermakova, A. O., Whiting, C. V., Trout, K., Clubbe, C., Fowler, N., \& Terry, M. K. (2020). Ecology and conservation of peyote in Texas, USA: Comparative survey of Lophophora williamsii populations in Tamaulipan Thornscrub and Chihuahuan Desert. bioRxiv. https://doi.org/10.1101/2020.04.03.023515.

Francuski, X. (2020). Is the world running out of Ayahuasca? [Internet]. EntheoNation. Available from: https://entheonation. $\mathrm{com} / \mathrm{blog} /$ ayahausca-sustainability-caapi-vine/?fbclid $=$ IwAR2f VXZnakWuthz_r2psNOPp4a8VOfRByQzreuxdrun9ksCkhidy YFMYX30.

Garcia-Romeu, A., Kersgaard, B., \& Addy, P. H. (2016). Clinical applications of hallucinogens: A review. Experimental and Clinical Psychopharmacology, 24(4), 229-268. https://doi.org/ 10.1037/pha0000084.

Gearin, A. K. (2017). Good mother nature: Ayahuasca neoshamanism as cultural critique in Australia. In Labate, B., Cavnar, C., \& Gearin A. K. (Eds.), The world Ayahuasca Diaspora: Reinventions and controversies (pp. 123-141). London: Routledge.

Gerber, K., Flores, I. G., Ruiz, A. C., Ali, I., Ginsberg, N. L., \& Schenberg, E. E. (2021). Ethical concerns about psilocybin intellectual property. ACS Pharmacology \& Translational Science. https://doi.org/10.1021/acsptsci.0c00171.

Hartogsohn, I. (2016). Set and setting, psychedelics and the placebo response: An extra- pharmacological perspective on psychopharmacology. Journal of Psychopharmacology, 30(12), 12591267. https://doi.org/10.1177/0269881116677852.
Harvey, I. (2020). A nuanced reflection of María Sabina by Ifetayo Harvey [Internet]. People of Color Psychedelic Collective. Available from: https://www.pocpsychedelics.com/blog.

Heidegger, M. (1977). The question concerning technology. New York \& London:Garland Publichsing Inc.

Hoffmann, W. A. (2015) Benefit-sharing. In ten Have, H. (Ed.), Encyclopedia of global bioethics. Cham: Springer. https://doi. org/10.1007/978-3-319-05544-2_38-1.

International Bioethics Committee. (2012). Report of the IBC on traditional medicine systems and their ethical implications. UNSESCO. Available from: https://unesdoc.unesco.org/ark:/ 48223/pf0000217457.

Labate, B. (2020). Psychedelic scientists should honor indigenous plants and traditions [Internet]. Chacruna Institute for Psychedelic Plant Medicines. Available from: https://chacruna.net/ psychedelic-scientists-indigenous-plants/.

Labate, B., \& Cavnar, C. (2014). Ayahuasca shamanism in the Amazon and beyond (pp. 171-177). Oxford Ritual Studies.

Lagarde, J. (2021). Spiritual tourism: The toxic impact on indigenous peoples and ethical steps forward as psychedelics boom [Internet]. Psychedelic Spotlight. Available from: https:// psychedelicspotlight.com/spiritual-tourism-toxic-impact-onindigenous-peoples-ethical-steps-forward-psychedelics-boom/.

Mackey, T. K., \& Liang, B. A. (2012). Integrating biodiversity management and indigenous biopiracy protection to promote environmental justice and global health. American Journal of Public Health, 102(6), 1091-1095. https://doi.org/10.2105/ AJPH.2011.300408.

MAPS. (2021). MAPS' phase 3 trial of MDMA-assisted therapy for PTSD Achieves successful results for patients with severe, chronic PTSD [Internet]. Multidisciplinary Association for Psychedelic Studies. Available from: https://maps.org/news/ media/9122-maps-phase-3-trial-of-mdma-assisted-therapy-forptsd-achieves-successful-results-for-patients-with-severechronic-ptsd.

Marseille, E., Kahn, J. G., Yazar-Klosinski, B., \& Doblin, R. (2020). The cost-effectiveness of MDMA-assisted psychotherapy for the treatment of chronic, treatment-resistant PTSD. Plos One, 15 (10), e0239997. https://doi.org/10.1371/journal.pone.0239997.

Miceli McMillan, R. (2020). Prescribing meaning: Hedonistic perspectives on the therapeutic use of psychedelic-assisted meaning enhancement. Journal of Medical Ethics. Published Online First: 04 November 2020. https://doi.org/10.1136/medethics2020-106619.

Milson, S. T., \& Pratt, M. (2017). How to sustainably harvest wild chaga mushrooms [Internet]. Black Magic. Available from: https://blackmagicalchemy.com/blogs/blog/how-tosustainably-harvest-chaga-mushrooms.

Móró, L., Simon, K., Bárd, I., \& Rácz, J. (2011). Voice of the psychonauts: Coping, life purpose, and spirituality in psychedelic drug users. Journal of Psychoactive Drugs, 43(3), 188-198. https://doi.org/10.1080/02791072.2011.605661.

Negrin, D. (2020). Colonial shadows in the psychedelic renaissance [Internet]. Chacruna Institute for Psychedelic Plant Medicines. Available from: https://chacruna.net/colonial-shadows-in-thepsychedelic-renaissance/.

Nickles, N., \& Nickles, D. (2014). When DMT equals killing the environment [Internet]. The Nexian. Available from: https:// 
the-nexian.me/home/knowledge/131-when-dmt-equals-killingthe-environment.

Psychedelic Community. (2018). How sustainable is the ever more popular use of Ayahuasca? [Internet]. Medium. Available from: https://medium.com/samadhi-today/sustainability-in-the-newera-of-ayahuasca-a77d97399605.

Ramirez Garcia H. S. (2014) Biopiracy. In ten Have, H. (Ed.), Encyclopedia of global bioethics. Cham: Springer. https://doi. org/10.1007/978-3-319-05544-2_57-1.

Reiff, C. M., Richman, E. E., Nemeroff, C. B., Carpenter, L. L., Widge, A. S., Rodriguez, C. I., et al. (2020). Psychedelics and psychedelicassisted psychotherapy. The American Journal of Psychiatry, 177(5), 391-410. https://doi.org/10.1176/appi.ajp.2019.19010035.

Roseman, L., Nutt, D. J., \& Carhart-Harris, R. L. (2018). Quality of acute psychedelic experience predicts therapeutic efficacy of psilocybin for treatment-resistant depression. Frontiers in Pharmacology, 8: 974. https://doi.org/10.3389/fphar.2017.00974.

Rosner, A. (2020). 2nd gen psychedelic drugs for depression can be safer for older adults [Internet]. Forbes. Available from: https:// www.forbes.com/sites/abbierosner/2020/06/15/2nd-genpsychedelic-drugs-for-depression-can-be-safer-for-olderadults/?sh $=4 \mathrm{a} 7177 \mathrm{~d} 22 \mathrm{fb} 3$.

Rucker, J. J. (2015). Psychedelic drugs should be legally reclassified so that researchers can investigate their therapeutic potential. BMJ, 350, h2902. https://doi.org/10.1136/bmj.h2902.

Siebert, A. (2021a). Better life pharma is developing Second generation psychedelics: Drugs that leave out the trip [Internet]. Forbes. Available from: https://www.forbes.com/sites/amandasiebert/ 2021/01/18/betterlife-pharma-is-developing-second-generationpsychedelics-drugs-that-leave-out-the-trip/?sh=6bb09f79648f.

Siebert, A. (2021b). Hamilton Morris on Mistakes, 5-MeO-DMT and preserving psychedelic toads [Internet]. Forbes. Available from: https://www.forbes.com/sites/amandasiebert/2021/01/08/ hamilton-morris-on-mistakes-5-meo-dmt-and-preservingpsychedelic-toads/?sh $=75 \mathrm{e} 7 \mathrm{ff} 3250 \mathrm{~b} 2$.

Smith, D. W. (2018). Phenomenology. In Zalta, E. N. (Ed.), The Stanford Encyclopedia of philosophy (Summer ed.). Stanford University: Metaphysics Research lab.

Smith W. R., \& Sisti D. (2020). Ethics and ego dissolution: The case of psilocybin. Journal of Medical Ethics. Published Online First: 27 May 2020. https://doi.org/10.1136/medethics-2020-106070.

Strauss, D., de la Salle, S., Sloshower, J., \& Williams, M. T. (2021). Research abuses against people of colour and other vulnerable groups in early psychedelic research. Journal of Medical Ethics Published Online First: 12 July 2021. https://doi.org/10.1136/ medethics-2021-107262.

Svenaeus, F. (2017). Phenomenological bioethics: Medical technologies, human suffering, and the meaning of being alive. Routledge, 75-96. https://doi.org/10.4324/9781315210131.

The Sabina Project. (n.d.). Mission [Internet]. Available from: https://www.thesabinaproject.com/mission.

Tomoski, M. (2019). What's lost when psychedelic mushrooms become synthetic Psilocybin [Internet]. Double Blind. Available from: https://doubleblindmag.com/synthetic-psilocybin-sabinawasson/.

Virdi, J. (2020). Indigenous voice in peyote conservation: Preserving medicine for future generations [Internet]. Psychedelics Today. Available from: https://psychedelicstoday.com/2020/09/ 16/indigenous-voices-in-peyote-conservation/.

Wasson, R. G. (1957). Seeking the magic mushroom. Life, 42(19), 100-120.

Weil, A. T., \& Davis, W. (1994). Bufo alvarius: A potent hallucinogen of animal origin. Journal of Ethnopharmacology, 41(1-2), 1-8. https://doi.org/10.1016/0378-8741(94)90051-5. 